\title{
Ethnobotanical study of plants used by Thami community in Ilam District, eastern Nepal
}

\author{
Krishna Ram Bhattarai \\ Department of Plant Resources, Kathmandu, Nepal \\ E-mail: krbhattarai@gmail.com
}

\begin{abstract}
An ethnobotanical survey was carried out on the utilization of plants by Thami communities in Gorkhe, Jogmai and Nayabazar areas of Ilam by interviewing traditional herbalists and different age groups of men and women in June 2016. A total of 30 plants belonging to 24 families and 29 genera have been documented. These plants were used for food, fodder, firewood, medicine and in rituals. The documented medicinal plants were used to treat various human ailments of 12 categories; with the highest number of species being used for gastro-intestinal disorders (15 spp.) followed by ENT problems (14 spp.). Most of the medicines were prepared from underground parts in the form of paste and used orally. Informant Consensus Factor $\left(F_{i c}\right)$ ranges from 0.6 to 0.9 with an average of 0.82 . Dermatological disorders have the highest $F_{i c}(0.90)$ and other categories have the lowest (0.6). Aconitum palmatum, Begonia picta, Bergenia ciliata, Astilbe rivularis, Swertia chirayita, Drymaria cordata and Remusatia pumila have the highest fidelity level (100\% each) and Galium asperifolium has the lowest FL (16\%). According to the use value, Swertia chirayita (UV=2.83) was the most important with uses against 6 ailments and Hypericum cordifolium has the least (UV=0.08), used in only one ailment. A variation in ethnobotanical knowledge was found according to age, gender and occupation in this community.
\end{abstract}

Key words: Ethnobotany, Informant consensus factor, Medicinal plants, Use value

DOI: http://dx.doi.org/10.3126/on.v16i1.22123

Manuscript details: Received: 24.09.2018 / Accepted: 16.11.2018

Citation: Bhattarai, K.R. 2018. Ethnobotanical study of plants used by Thami community in Ilam District, eastern Nepal, Our Nature 16(1): 55-67. DOI: http://dx.doi.org/ 10.3126/on.v16i1.22123

Copyright: (C) Bhattarai 2018. Creative Commons Attribution - Non Commercial 4.0 International License.

\section{Introduction}

Traditional beliefs about the diverse uses of plants are deeply rooted in Nepalese culture. The various ethnic groups of the country have developed their own knowledge systems for the use of plants in food, clothing, shelter, medicine and their spiritual needs (Rajbhandari and Wrinkler, 2015). From time immemorial many medicinal plants are well known in Nepal for various ailments (Bhattarai and Basukala, 2016). Therefore, country was mentioned as a sacred heaven of medicinal and aromatic plants in Vidic and Pauranic literature (Baral and Kurmi, 2006), which founded the base of the Ayurvedic system of medicine (Bajpai et al., 2016). Plants are the most important source of traditional medicines throughout the world (Bhattarai, 1989) and are the source of many major pharmaceutical drugs (Sarwar et al., 2011). At present, about 30,000 to 70,000 plant species are using medicinally across the world and $70 \%$ of the world's rural people depending upon such plants for their primary health care (WHO, 2002). In Nepal, a total 1950 species of plants are found to be medicinal of which 1614 species are native (Ghimire, 2008) and much more yet to be explored (Manandhar, 2002). Baral and Kurmi (2006) reported 1792 
plant species of medicinal uses comprising indigenous, endemic, cultivated, exotic and naturalized taxa.

Nepal is a multi-ethnic, multi-lingual and multi-cultural country where, 26.5 million people, under 125 caste or ethnic groups speak 123 different kinds of languages (CBS, 2013). Thami is one of the 59 communities officially recognized as indigenous nationalities (HMG, 2002), whose settlements are centered in Tamakoshi area and northern part of Dolakha District (Budathoki et al., 2008). The majority of them live in Dolakha, Sindhupalchok and Ramechap Districts, with notable smaller populations in Ilam, Jhapa, Udayapur and Bhojpur Districts (Shneiderman and Turin, 2006). There were 28671 individuals of Thami (CBS, 2013), of which 912 live in Ilam (CBS, 2014a). There is also Thami community in north-east India (Darjeeling and Sikkim), Tibet and Bhutan (Shneiderman and Turin, 2000, 2006; URL, 2018 a, b). Thami is a Nepali term used by other castes in place of Thangmi means 'people of pasture land' or 'people living in border land' (Shneiderman and Turin, 2000). They speak Tibeto-Burman language having lexical similarities with Newari language (Turin, 2004). They were originally a nomadic tribe. In the past, they had ample land areas under Kipat land. Later on, other castes immigrated and encroached over their land. Their main occupation is agriculture and livestock rearing. They also engage in stone quarrying, masonry, carpentry, bamboo work, weaving bhangra and colleting herbs. Very few people are involved in driving, local business, teaching and foreign employment. Their main religion is Hinduism, followed by Buddhism, Animist, and Kiranti (Budathoki et al., 2008), and many people are diverted to Christianity (URL, 2018 a, b). Udhauli, Uvauli, Baisakh Purnima, Dashain, Tihar, Janai purnima, Maghi sankrati etc. are some main festival of Thami people (Budathoki et al., 2008; Thami, 2017).

In recent years, there are many studies related to medicinal plants and associated indigenous knowledge in Nepal (Baral and Kurmi, 2006), but these studies less emphasized to highly marginalized groups including Thami. Till the date, there is only one ethnobotanical documentation related to Thami communities in Dolakha District of Nepal conducted by a British linguist and anthropologist, Dr. Mark Turin. While documenting the grammar of Thami language, Mark Turin listed 127 plants with their uses in agriculture, dye, furniture, construction, medicine, fish poison, fodder, food or in cultural and ceremonial occasions (Turin, 2003). Present study basically focused on the documentation of medicinal use of plants, their preparation and application in Thami community of Gorkhe area of Suryodaya Municipality, and Jogmai and Nayabazar areas of Maijogmai rural municipality of Ilam District. This study provides an inventory of medicinal plants used in Thami community against different human ailments.

\section{Materials and methods Study area}

Ilam (Lat. $26^{\circ} 40^{\prime} \mathrm{N}-27^{\circ} 08^{\prime} \mathrm{N}$, Long. $87^{\circ} 40^{\prime} \mathrm{E}-$ $88^{\circ} 10^{\prime} \mathrm{E}$, area $1,703 \mathrm{~km}^{2}$ ) is a hilly district situated in the eastern region of Nepal in Province number 1 . The district stretches from lower belt of terai and chure to the upper hilly belt of the Himalayan region with altitude ranging from $140 \mathrm{~m}$ to $3636 \mathrm{msl}$. The average annual temperature is $20.5^{\circ} \mathrm{C}$ and the average annual rainfall is $2500 \mathrm{~mm}$. The tropical to alpine vegetation is found in the district with forest coverage of about 55\% (DFRS, 2015). There are 912 individuals of Thami residing in Jogmai $(n=453)$, Nayabazar $(n=164)$, Gorkhe $(\mathrm{n}=161)$, Phikkal $(\mathrm{n}=60)$, Pasupatinagar $(\mathrm{n}=47)$, Ilam Municipality $(n=14)$ and Sri Antu $(n=13)$ (CBS, 2014b). Jogmai, Nayabazar (Maijogmai rural municipality) and Gorkhe (Suryodaya municipality) areas were selected as study sites because highest number of Thami people found to live there. These study sites are located in the hilly area of north-east part of Ilam (Fig. 1).

\section{Selection of informants}

Prior to documentation of ethnobotanical information, first a meeting was organized in Jogmai with of pre-informed people from Jogmai, Gorkhe and Nayabazar areas. In that gathering, various medicinal plants available and used in their community were listed and collected. After gathering the collected plants, 30 specimens were selected randomly to document detail information. Among the participants, 12 people (Males $=6$, Females $=6$ ) of three age groups $(20-40, n=7 ; 40-60, n=3$; and $60+, n=2)$ with different occupations were selected inclusively as key informants to compare their ethnobotanical knowledge. Each of them was provided to fill up 30 sheets of questionnaire, each sheet for individual plant.

\section{Data collection}


The ethnobotanical data were collected in June 2016 by using structured and semi-structured questionnaire with key informant interview. Twelve key informants were interviewed by showing the fresh plant specimens that were collected by them. Questionnaire survey was carried out in order to compare and analyze the knowledge among the informants about the habit

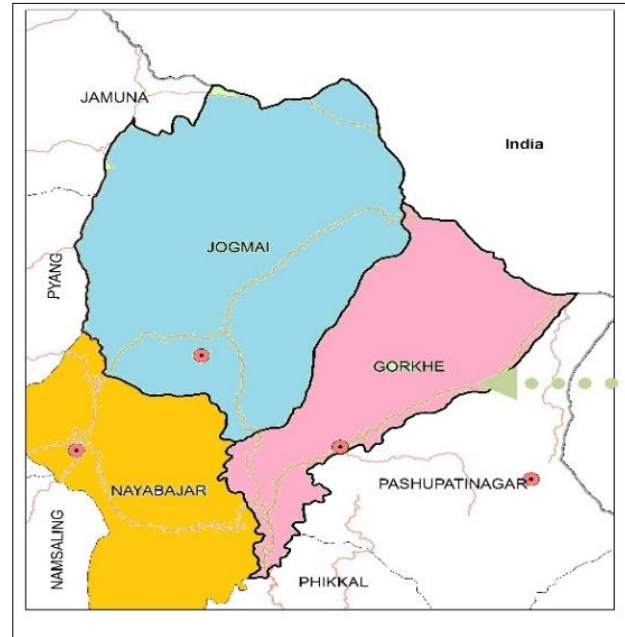

Figure 1. Map of study area and habitat of plant, flowering period, local status, threat, cultivation practice, trade, uses, medication forms, dose and route of administration of medicines etc. Reported ailments were grouped into major categories following Heinrich et al. (1998).

The collected plant specimens were photographed, pressed in between newspapers and dried in the field using a natural drying technique in sunlight (Forman and Bridson, 1989). Scientific names were determined by using different books (Polunin and Stainton, 1984; Stainton, 1988; Shrestha, 1998; Manandhar, 2002; Baral and Kurmi, 2006). The nomenclature of APG III was followed (www.theplantlist.org). Voucher specimens were deposited at the herbarium of Plant Research Centre, Ilam (formerly known as District Plant Resources Office, Ilam).

\section{Data analysis}

(1) Informant Consensus Factor $\left(F_{i c}\right)$

The level of homogeneity among information provided by different informants was calculated by the Informant Consensus Factor $\left(F_{i c}\right)$ according to Heinrich et al. (1998) as:

$F_{i c}=\left(N_{u r}-N_{t}\right) /\left(N_{u r}-1\right)$,

Where, $N_{u r}=$ Number of use citations in each ailment category, $N_{t}=$ Number of species used

(2) Fidelity Level (FL)

The fidelity level $(F L)$ determines the most frequently used plant species for treating a

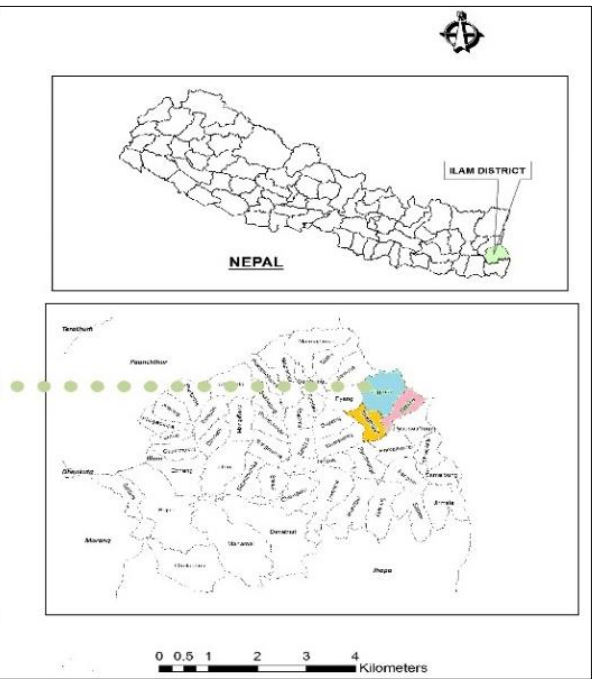

particular ailment category by the informants. The FL was calculated following Friedman et al. (1986) as:

$F L(\%)=\left(N_{p} / N\right) \times 100$

Where, $N_{p}=$ Number of informants that reported a use of a plant against a particular disease category, and $N=$ Total number of informants that used the plants against any given disease.

(3) Use Value (UV)

The relative importance of ethnomedicinal plant species was calculated by using the use value (UV) for each species (Phillips and Gentry, 1993).

$U V_{s}=\left(\sum U_{s}\right) /\left(N_{s}\right)$

Where $U_{s}=$ Total number of use-reports cited by each informant for a given plant species $s$ and $N_{s}$ $=$ Total number of informants interviewed for plant species $s$.

\section{Results and discussion \\ Plant diversity and uses}

Of the randomly selected 30 plants (24 families and 29 genera), 28 were dicots and 2 monocots. These were represented by highest numbers of herbs $(n=19)$ followed by trees $(n=6)$, climbers $(n=3)$ and shrubs $(n=2)$. The dominant family was Rutaceae (with 3 species), followed by Apiaceae, Ranunculaceae, Rosaceae and Sexifragaceae (with 2 species each). Rest of the 19 families had one plant each (Table 1). The study showed that different parts of the same plants are used for different purposes (food, 


\begin{tabular}{|c|c|c|c|c|c|c|c|c|c|c|c|c|}
\hline 它 & & 䏓 & 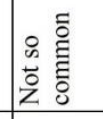 & 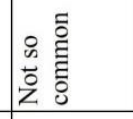 & 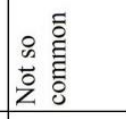 & 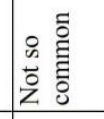 & 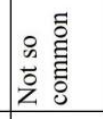 & ठ̃ & 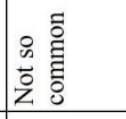 & . & 峁 & 吾 \\
\hline 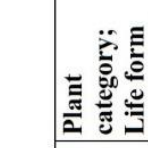 & & 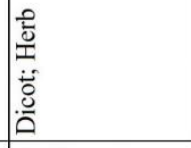 & 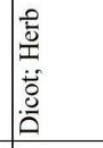 & 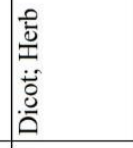 & 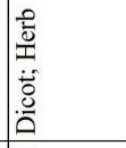 & 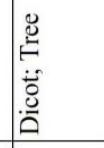 & 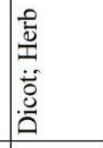 & 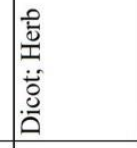 & 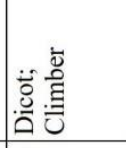 & 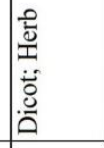 & 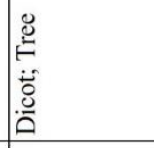 & 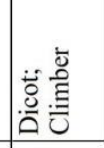 \\
\hline 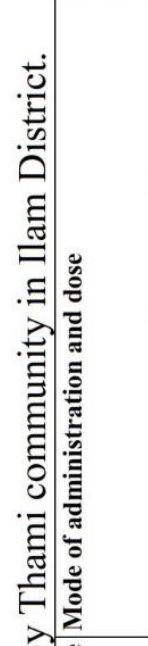 & 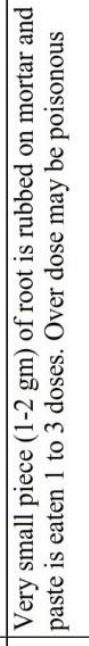 & 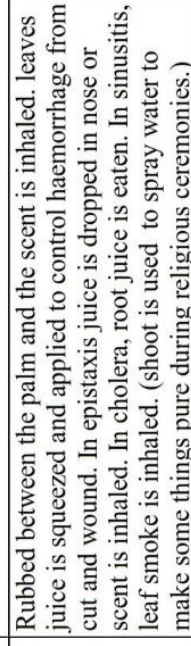 & 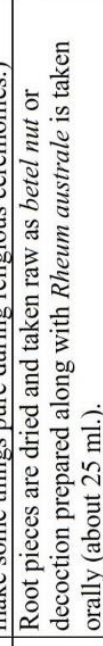 & 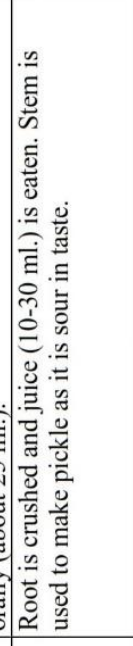 & 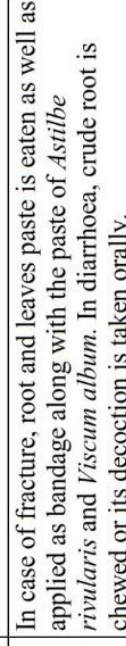 & 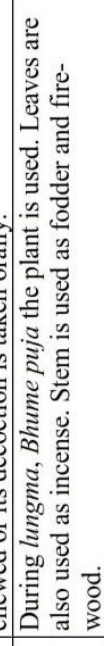 & 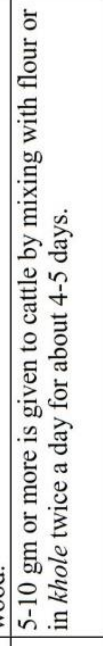 & 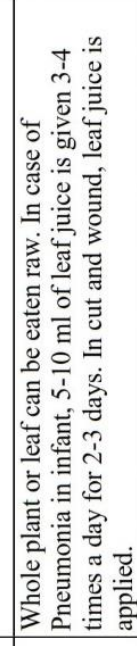 & 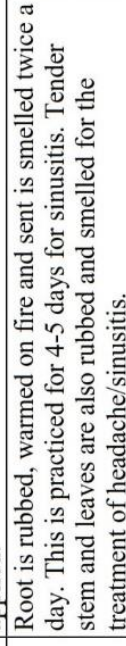 & 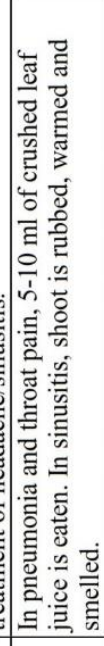 & 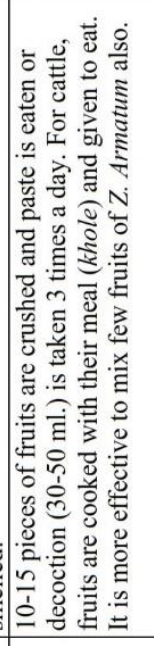 & 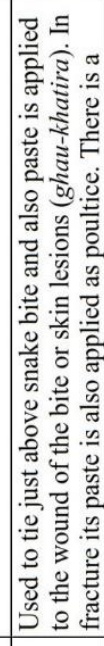 \\
\hline 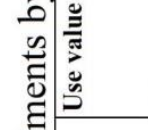 & तิ & $\stackrel{m}{?}$ & $\stackrel{\infty}{?}$ & 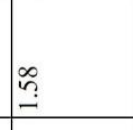 & $\bar{\sigma}$ & 。 & 0 & $\underset{i}{\infty}$ & 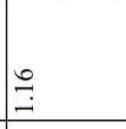 & $\overline{2}$ & $\hat{\imath}$ & $\bar{\delta}$ \\
\hline 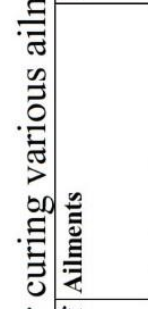 & 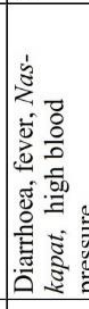 & 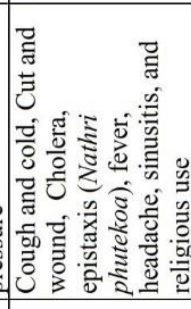 & 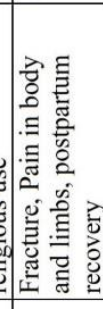 & 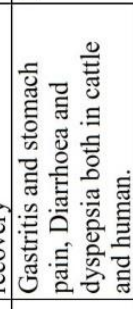 & 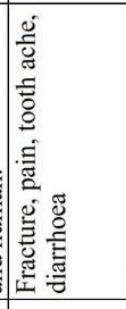 & 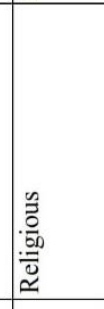 & 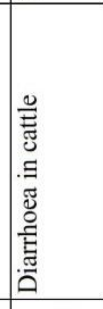 & 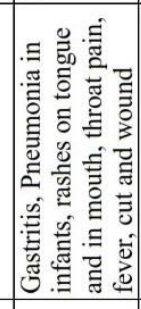 & 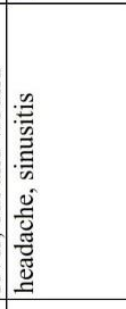 & 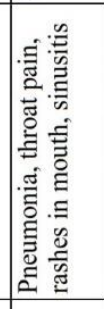 & 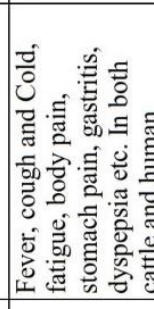 & 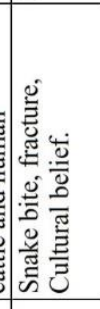 \\
\hline 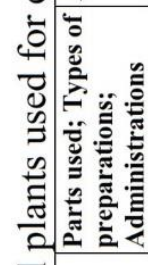 & 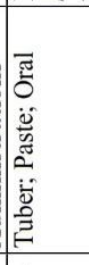 & 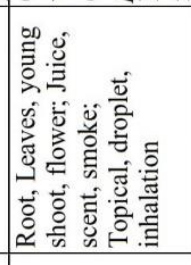 & 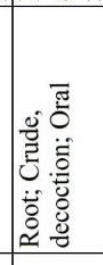 & 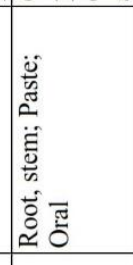 & 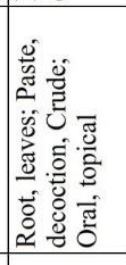 & 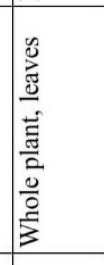 & 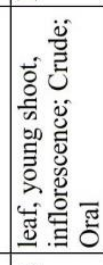 & 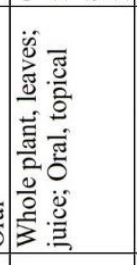 & 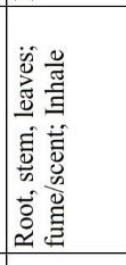 & 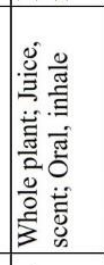 & 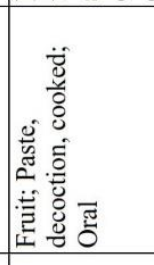 & 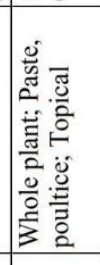 \\
\hline 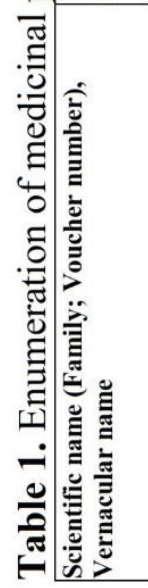 & 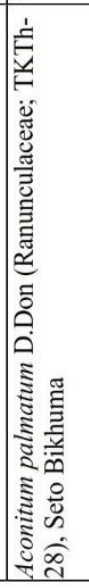 & 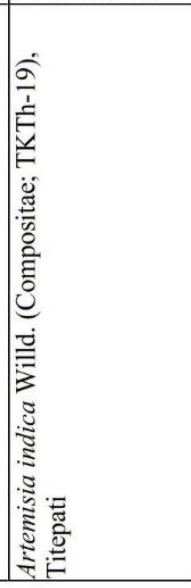 & 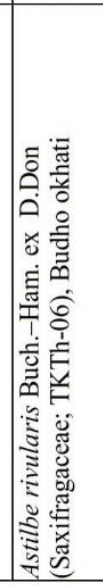 & 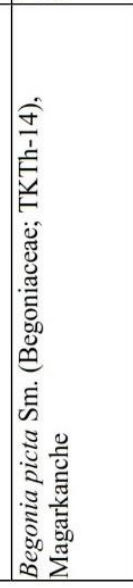 & 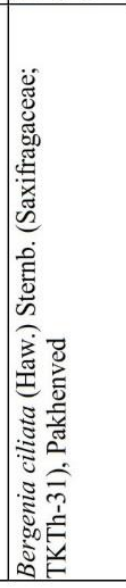 & 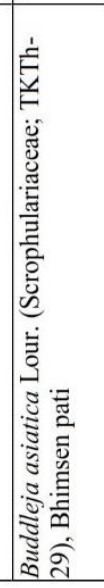 & 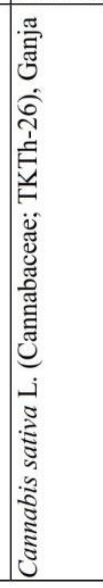 & 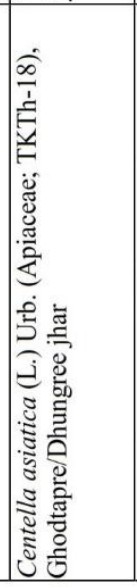 & 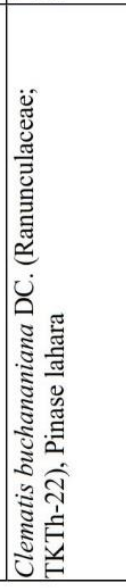 & 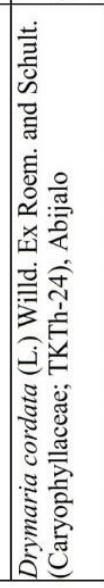 & 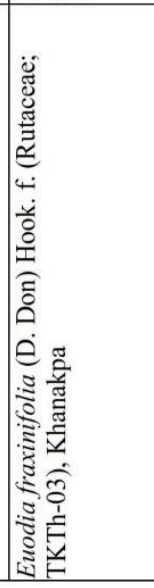 & 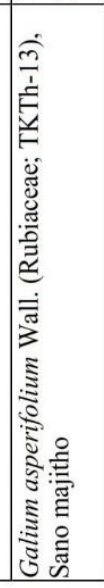 \\
\hline
\end{tabular}




\begin{tabular}{|c|c|c|c|c|c|c|c|c|c|c|}
\hline & \begin{tabular}{|l} 
\\
है \\
童 \\
\end{tabular} & 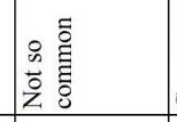 & 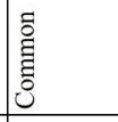 & 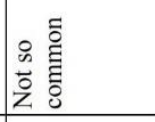 & 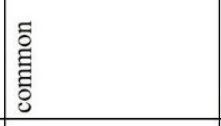 & 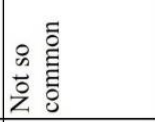 & 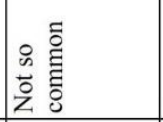 & 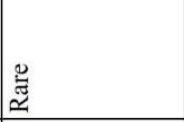 & 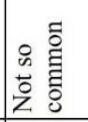 & 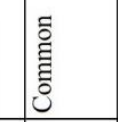 \\
\hline & 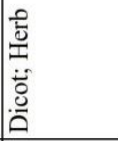 & 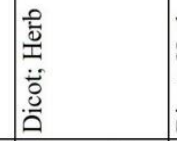 & 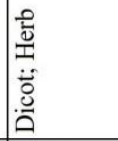 & 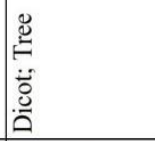 & 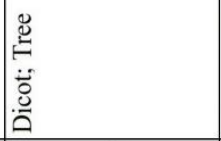 & $\begin{array}{l}I \\
\ddot{z} \\
\ddot{0} \\
\ddot{0}\end{array}$ & 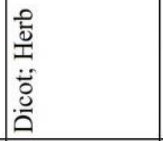 & 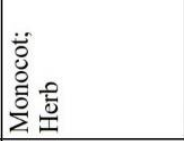 & 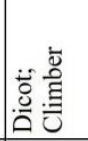 & 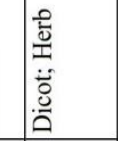 \\
\hline 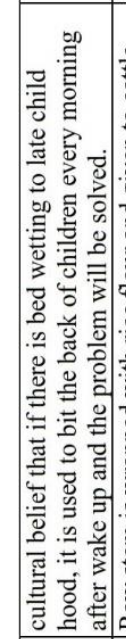 & 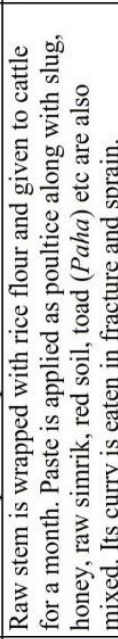 & 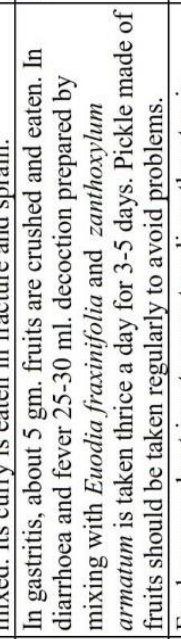 & 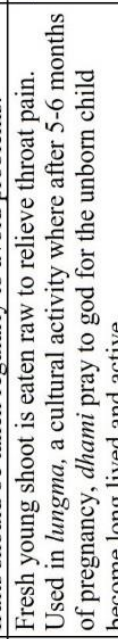 & 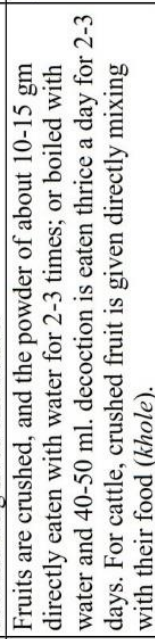 & 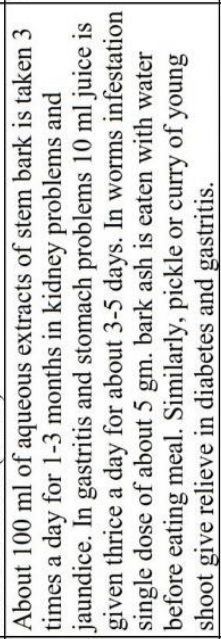 & 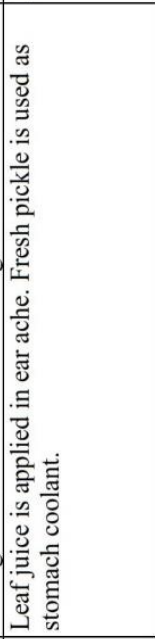 & 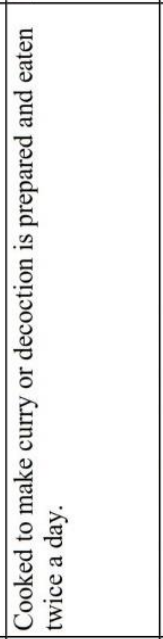 & 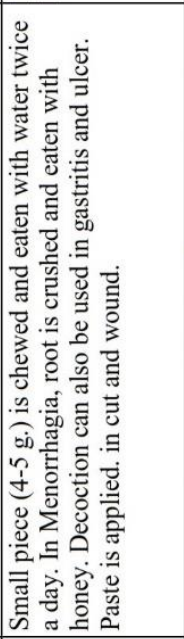 & 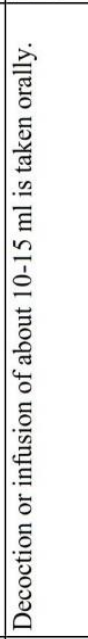 & 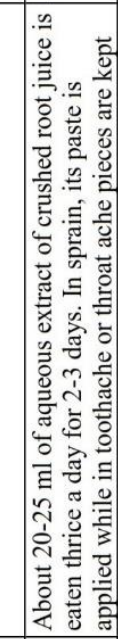 \\
\hline & 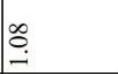 & 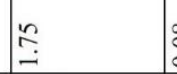 & 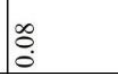 & $\alpha$ & 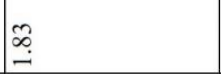 & $\bar{\Omega}$ & $\overline{9}$ & 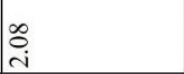 & 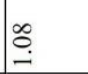 & $\bar{\Omega}$ \\
\hline & 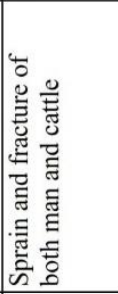 & 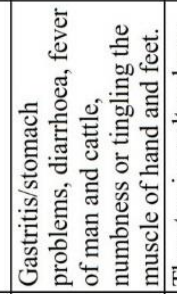 & 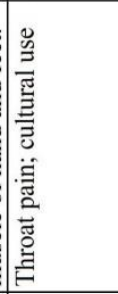 & 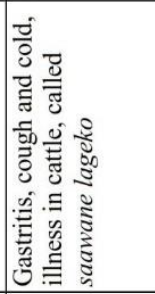 & 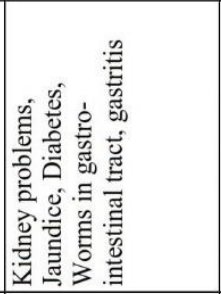 & 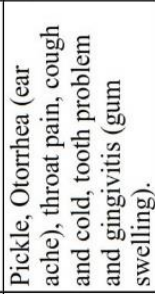 & 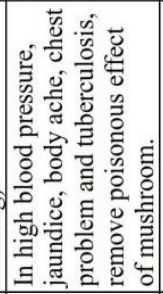 & 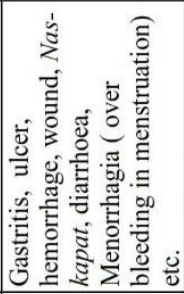 & 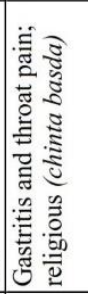 & 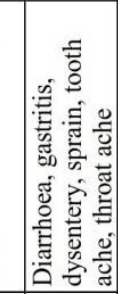 \\
\hline & 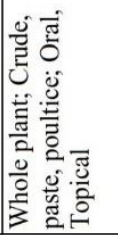 & 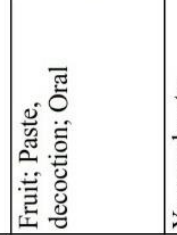 & 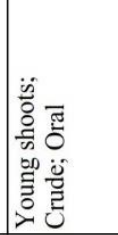 & 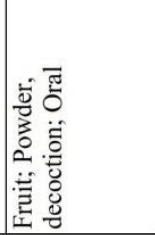 & 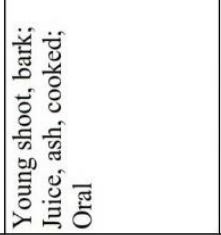 & 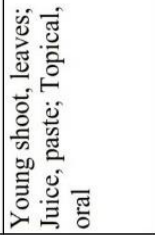 & 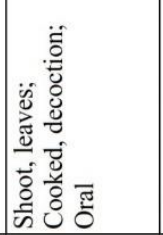 & 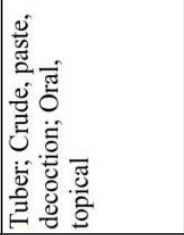 & 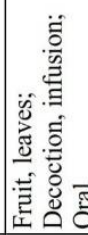 & 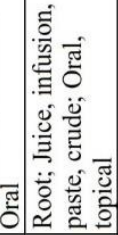 \\
\hline & 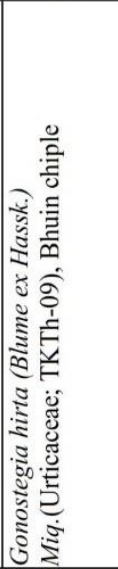 & 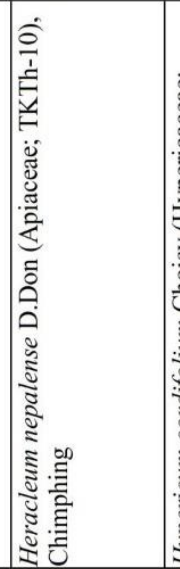 & 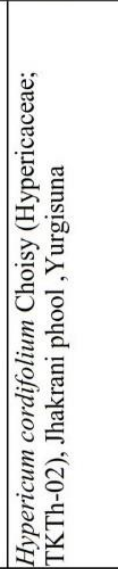 & 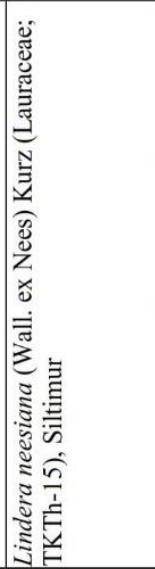 & 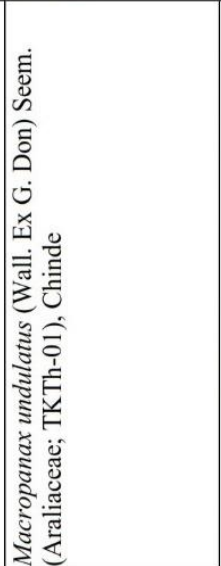 & 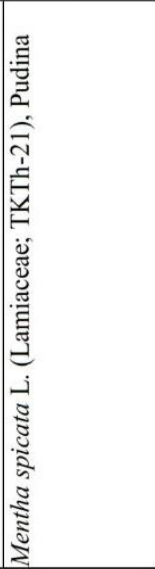 & 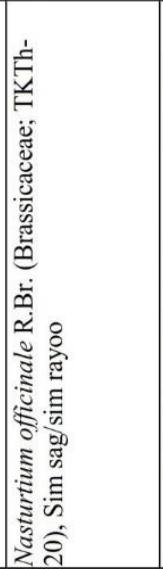 & 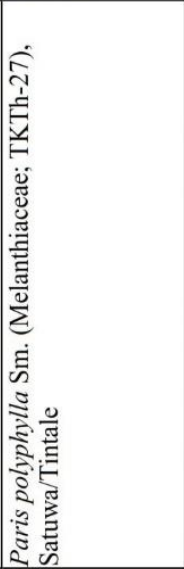 & 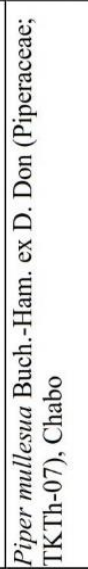 & 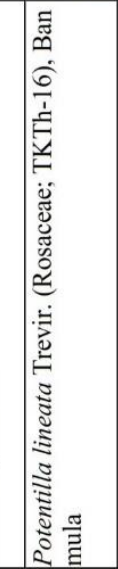 \\
\hline
\end{tabular}




\begin{tabular}{|c|c|c|c|c|c|c|c|}
\hline 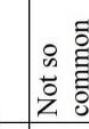 & & 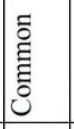 & 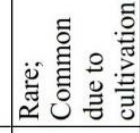 & 递 & 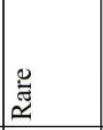 & 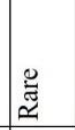 & 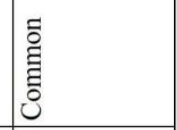 \\
\hline 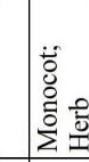 & 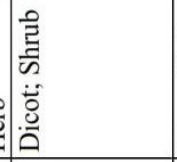 & 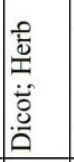 & 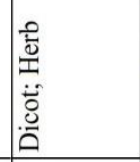 & 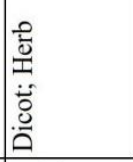 & 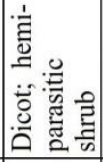 & 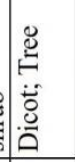 & 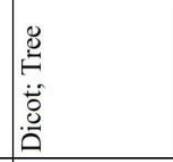 \\
\hline 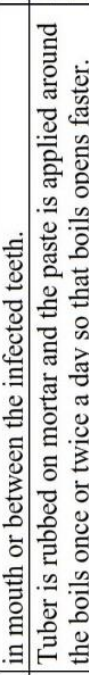 & 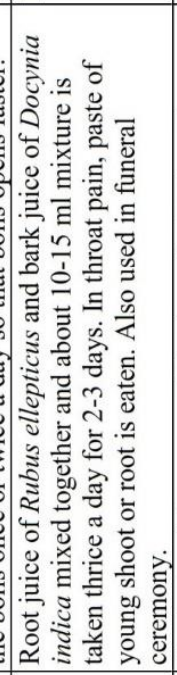 & 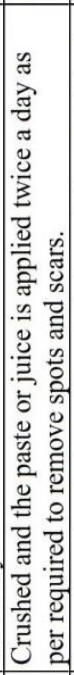 & 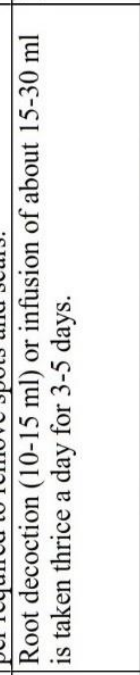 & 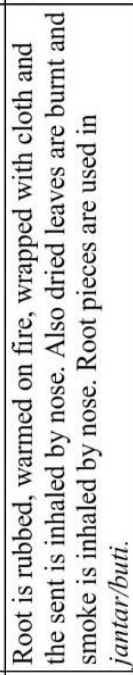 & 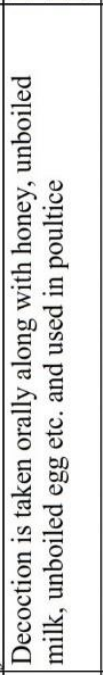 & 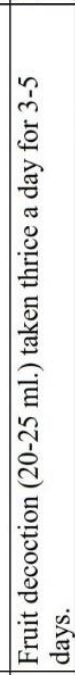 & 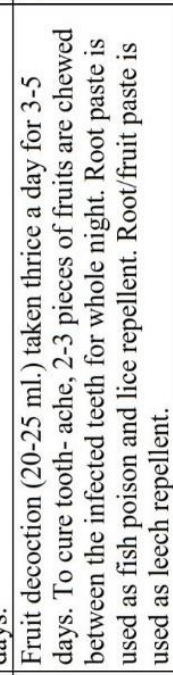 \\
\hline$\stackrel{\infty}{\circ}$ & 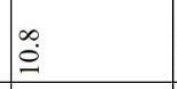 & $\stackrel{\overbrace{}}{\overbrace{}}$ & 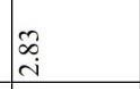 & ઼ָิ & - & : & $\stackrel{\infty}{\infty}$ \\
\hline 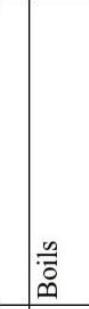 & 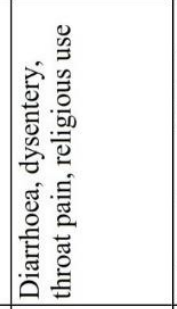 & 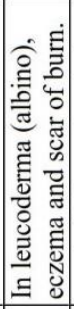 & 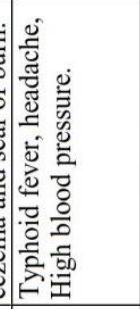 & 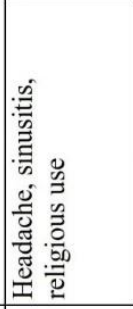 & 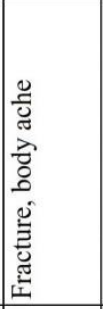 & 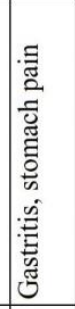 & 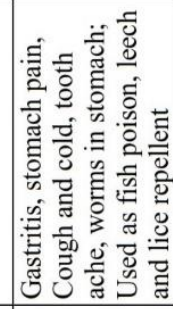 \\
\hline 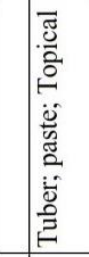 & 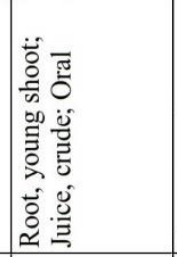 & 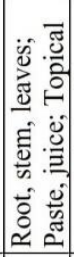 & 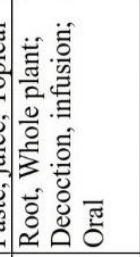 & 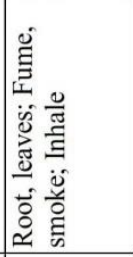 & 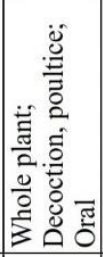 & 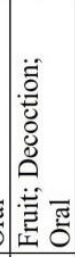 & 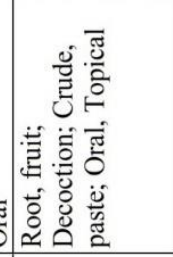 \\
\hline 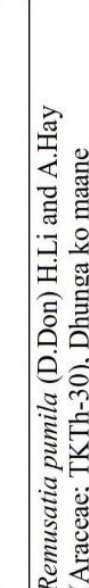 & 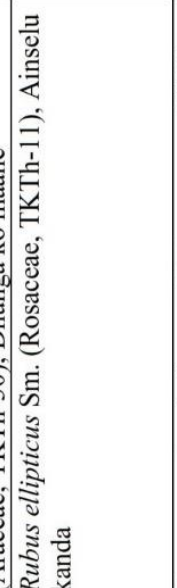 & 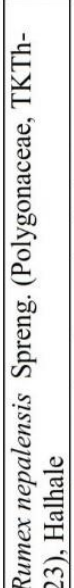 & 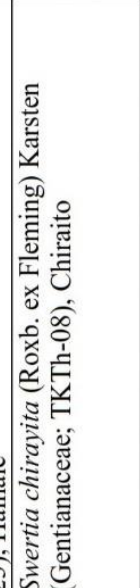 & 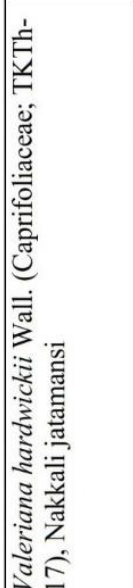 & 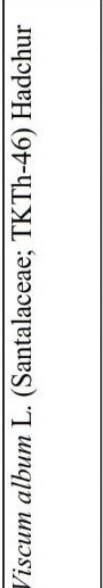 & 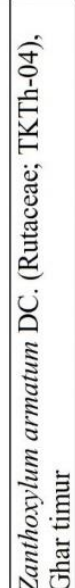 & 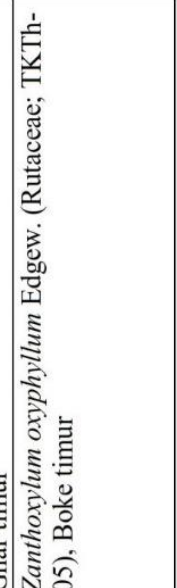 \\
\hline
\end{tabular}


fodder, fuel wood etc.) and for different aliments. Among 30 plant species, Buddleja asiatica was found to be used only in rituals and Cannabis sativa had reported to treat only cattle ailment. Remaining 28 species were reported to treat human ailments and some had additional uses. Apart from medicine, there were 3 plant species (Heracleum nepalense, Lindera neesiana and Zanthoxylum oxyphyllum) used to give flavour in locally prepared alcohol, 2 species (Macropanax undulates, Nasturtium officinale) as vegetables, 3 species (Begonia picta, Heracleum nepalense and Mentha spicata) as pickle, one species (Rubus ellipticus) as wild fruit and one species (Euodia fraxinifolia) as making cover (Daap) of national weapon (Khukuri). In addition to this, 5 species (Artemisia indica, Hypericum cordifolium, Piper mullesua, Rubus ellipticus and Valeriana hardwickii) were used in Thami rituals and 5 species (Begonia picta, Euodia fraxinifolia, Gonostegia hirta, Heracleum nepalense, Lindera neesiana) were used against cattle ailments. It was found that Aconitum palmatum, Lindera neesiana and Swertia chirayita had high market value. The young shoots of Macropanax undulates commonly used as vegetable, so it could be sold in local market as well as international market (Darjeeling). The threat was found more in plants with multiple use value. These species with high use values are likely to be more vulnerable because of high demand and high collection pressure (Shrestha et al., 2014). Some potential threats to medicinal plants identified in this study were unsustainable harvesting, habitat destruction, deforestation, illegal trade and loss of spring-water resources and wetlands. The questionnaire results showed that some medicinal plants (eg. Macropanax undulates, Swertia chirayita) were threatened due to over collection and grazing.

Based on information collected from the informants, all the human ailments were grouped into 12 categories. The highest number of plants was used against digestive system disorders (15 spp.) followed by ENT problems (14 spp.), fever and headache (11 spp.) and so on (Fig. 2). The scenario of having plant used mainly for gastrointestinal disorders showed that there is enormous importance of this group of illnesses and may be the more frequent exchange of information for treating this ailment category (Heinrich et al., 1998). This is similar to the findings of several studies in rural part in Nepal (Singh et al., 2012; Thapa et al., 2013; Luitel et al., 2014; Shrestha et al., 2014; Bhattarai and Acharya, 2015; Bhattarai, 2017). To avoid such ailments, people should have good sanitation practices and supplied with safe drinking water (Rokaya et al., 2014). The uses of plants reported by the informants were compared with previous studies from the same ethnic group (Turin, 2003) and from various studies of the same region (Tamang and Singh, 2014; Bhattarai and Khadka, 2016; Subba et al., 2016) showed that there were many similar use reports of the documented plants. This shows that their pharmacological effectiveness is highly reliable (Giday et al., 2009) along with cultural influence and belief because traditional knowledge is influenced by ancestry, inter-cultural diffusion and interaction with natural environment (SaslisLagoudakis et al., 2014).

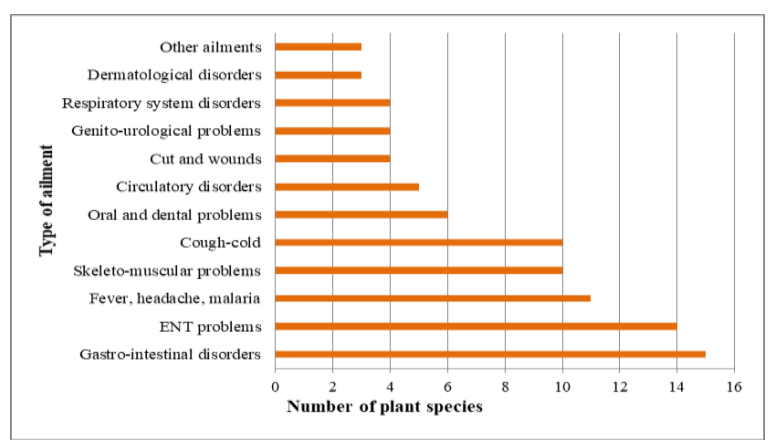

Figure 2. Number of medicinal plants used against different ailment categories

\section{Parts used, preparations, mode of administrations and harvesting}

Underground parts (roots/tubers) and leaves were the most frequently used plant parts followed by stem, fruits and flower/inflorescence (Fig. 3). The leaves, underground parts, seeds and fruits contain the high amount of biologically active substances compared to other parts (Srithi et al., 2009). The frequent use of underground parts or fruits/seeds showed that the plant species are likely to face threat in the future as they are most important parts for regeneration (Ghimire et al., 2008). It is thus important that cultivation techniques ought to be introduced in the areas to save the plant species and economic benefit of the community (Pradhan and Badola, 2008; Rokaya et al., 2010). The most frequent forms of preparation were paste followed by decoction (Fig. 4). The most common mode of administrations was oral followed by topical applications (Fig. 5). Plant species that were used as medicine mainly collected from the wild i.e. from private or community forest. One 
species (Swertia chirayita) was cultivated commercially. Few species (Heracleum nepalense, Mentha spicata, Macropanax undulates, Lindera neesiana) were domesticated either in their home garden or in the farm land. High valued medicinal plants were generally collected on the special day called harelo, first Tuesday after a Hindu festival-Teej, which falls in the month of August-September. It is believed that the medicinal plants collected on that day have good effects on the medicine which is similar to the ethnic community of Lepcha of Ilam (Bhattarai, 2017). Generally fresh parts of plants are used as medicine and all the informants agreed that the medicinal plants should be preserved for future.

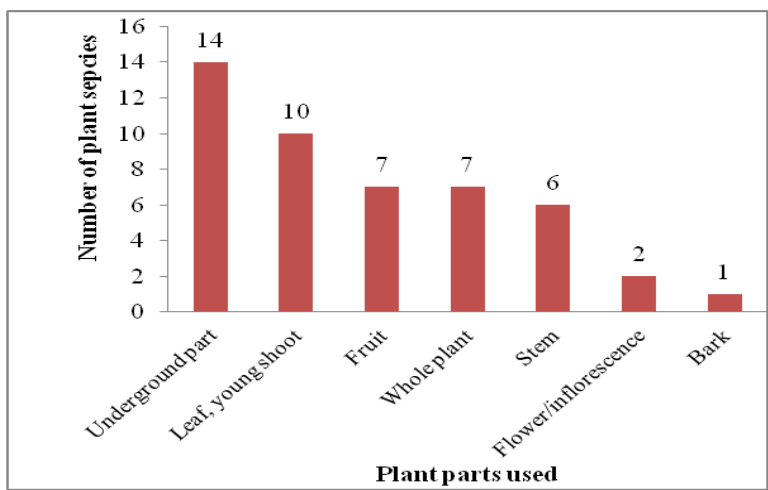

Figure 3. Different plant parts used for medicinal purpose

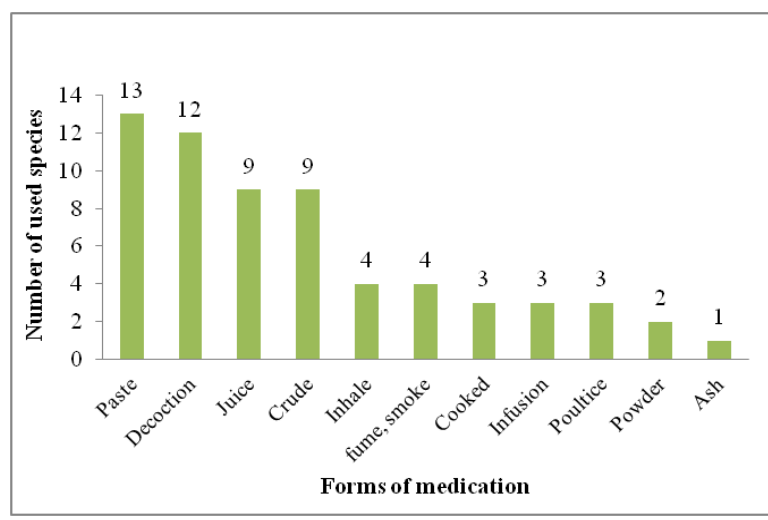

Figure 4. Medication forms used by Thami community

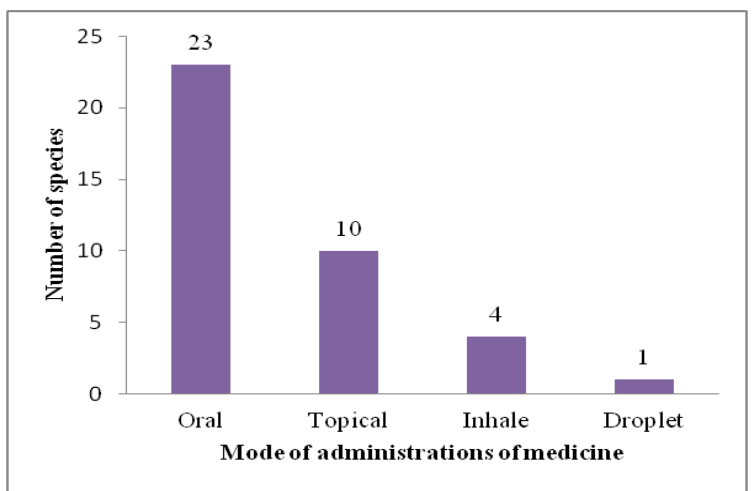

Figure 5. Mode of administrations of the medicine
Informant consensus factor, fidelity level and use value

The results of the informant consensus factor $\left(F_{i c}\right)$ showed that the value ranges from 0.6 to 0.9. The dermatological disorder has the highest $F_{i c}$ value of 0.90 with 32 use reports for 3 species. It is followed by gastro-intestinal disorder (134 use reports, 15 spp.). The others category (heat illness, antidote of mushroompoisoning and lice repellent) has the lowest $F_{i c}$ value 0.6 with 6 use reports for three plant species (Table 2). The high $F_{i c}$ value (close to 1) indicates that relatively few species are used by a large proportion of the healers (Heinrich et al., 1998). In this study the average $F_{i c}$ value for all ailment categories was found 0.82 , indicating a high level of agreement among the informants. This result is similar with the study in Rasuwa District, central Nepal (Uprety et al., 2010; Shrestha et al., 2014) and Rupandehi and Palpa District, Western Nepal (Singh et al. 2012; 2018), but different form the study in Humal District, western Nepal (Rokaya et al., 2010).

While selecting the most preferred plant species for each ailment category, the highest Fidelity level $(F L)$ values were considered in each category of ailment. Begonia picta and Aconitum palmatum ( $\mathrm{Fl}=100 \%$, each) for gastrointestinal ailments. Clematis buchananiana $(F L=$ 91.6\%) in ENT problems, Astilbe rivularis and Bergenia ciliata $(F L=100 \%$ each) in skeletomuscular problems, and so on (Table 3 ). The plant species with the highest $F L$ value is considered the most preferred and important species for a particular purpose (Hoffman and Gallaher, 2007). The fact that the plants with highest $F L$ values could be an indication of their good healing potential in their respective illness categories.

According to use value, Swertia chirayita $(U V=2.83)$ was the most important with 34 uses reports from 12 informants. It was followed by Euodia fraxinifolia $(U V=2.33)$ with 28 use reports and Aconitum palmatum $(U V=2.25)$ with 27 use reports. The species with least importance in medicine were Valeriana hardwickii $(U V=0.25)$ with 3 use reports and Hypericum cordifolium ( $U V=0.08)$ with single use report from 12 informants (Table 1). The highest use value of Swertia chirayita $(U V=2.83)$ showed that it is the most preferred species in this community to treat fever, headache and malaria, and lowest of Hypericum cordifolium (0.08), which means people use alternatives of this species to treat ENT problems. Plant species 
with low use values should not be ignored because failing to declare the importance of this plant to upcoming generations could decline the traditional knowledge (Mahmood et al., 2012).

\section{Comparison of ethnomedicinal knowledge according to age, sex and occupation}

The socio-economic level, age, gender and profession, are some of the variables that may influence the distribution of the knowledge about the uses of plant within a community (Toledo et al., 2009). The result showed that the age groups within the range $60^{+}$found more knowledgeable and reported highest uses (39\%) than the younger age group 40-60 (34\%) and 20-40 (27\%) (Table 4). Results of several studies conducted in different parts of the world (Giday et al., 2009) as well as Nepal (Luitel et al., 2014; Bhattarai and Tamang, 2017) revealed similar findings. This may be because the older generations of this community tend to function as keepers of traditional knowledge, they may know more traditional remedies and grew up with little or no exposure to modern health practices (Quinlan and Quinlan, 2007). Further, older individuals have had additional learning time to know about potential of plants and more exposure to illness events, treatments, and their outcomes than the younger generation. Women are predicted to be familiar with more herbal medicines than males. By the gender wise comparison, females documented slightly more use reports $(50.3 \%)$ than males $(49.6 \%)$. This may be because they are homemakers; they have to collect food, fodder, firewood and are responsible for the health of the family (TorresAvilez et al., 2016) and their cattle. Similar findings are reported in Mexico (BeltranRodriguez et al., 2014), Nepal (Luitel et al., 2014), Brazil (Meretika et al., 2010) and in Ethiopia (Giday et al., 2009). On the basis of occupation, there were 3 primary school teachers, 7 farmers and 2 traditional healers. The traditional healers were found most knowledgeable (use report=41\%) than farmers (use reports $=32 \%$ ) and primary school teachers (use reports $=27 \%$ ) because they have assimilated the knowledge about the medicinal plants in their traditional health care system and they do practice it in their day to day life (Bisht et al., 2006) as their profession.

The secrecy of ethnomedicinal knowledge is a common practice (Giday et al., 2009) and traditional healers hardly share their knowledge to outsiders with the belief that effectiveness would decrease if knowledge is reveled (Shrestha et al., 2014). This secrecy was also reported from the Humla District of western Nepal (Rokaya et al., 2010), Makwanpur District of central Nepal (Luitel et al., 2014) and Ilam District of eastern Nepal (Bhattarai, 2017). Nonsharing attitude is one of the strongest reasons for the depletion of traditional knowledge as pointed out by Pradhan and Badola (2008). The distinct pattern of modernization such as education, commercial occupation, acculturation etc. may help in erosion of traditional knowledge (Quinlan and Quinlan, 2007). The depletion is further accelerated by wider use of modern medicine and lack of successor of faith healers (Manandhar and Chaudhary, 1992) because successors are easily influenced by modernization (Bhattarai, 2017). The process of knowledge loss is further magnified by ruralurban migration. The next reason could be due to the depletion of plant resources as there are increasing human induced activities such as construction of roads, deforestations, fire, shifting cultivation, etc. Thus, it is obvious that there is degradation of language, culture and tradition in Thami community and in turn ethnobotanical knowledge is eroding. Additionally, this knowledge becomes danger if written documentation is insufficient or unavailable (Rani et al., 2017). Thus, the present work would help to preserve ethnobotanical knowledge of Thami community as communication between indigenous community with scientific community help to preserve traditional knowledge (Subba et al., 2016) through documentation and dissemination.

The few species were either highly preferred or have multiple uses in the study area. Some of the plant species fall in threat categories of IUCN (Bergenia ciliata), and IUCN and CAMP (Aconitum sp., Paris polyphylla, Swertia chirayita). Moreover, some threatened species are highly preferred over all the species such as Swertia chirayita $(U V=2.83)$ Euodia fraxinifolia $(U V=2.33)$, Aconitum palmatum $(U V=2.25)$ and Paris polyphylla $(U V=2.08)$. Therefore, such species should be prioritized for cultivation and sustainable management in order to ensure their long term availability (Shrestha et al., 2014). Commercial cultivation of Swertia chirayita and in-situ conservation of Paris Polyphylla was already started in the study area and further, Bergenia ciliata should also be cultivated. This will reduce pressure on these species in their natural environments as well as provide 
Table 2. Informants Consensus Factor $\left(F_{i c}\right)$ by categories of diseases.

\begin{tabular}{lccc}
\hline Disease categories & No. of species $\left(\boldsymbol{N}_{\boldsymbol{t}}\right)$ Use reports $\left(\boldsymbol{N}_{\boldsymbol{u}}\right)$ & Informants consensus factors $\left(\boldsymbol{F}_{\boldsymbol{i c}}\right)$ \\
\hline Dermatological disorders & 3 & 32 & 0.9 \\
\hline Gastro-intestinal disorders & 15 & 134 & 0.89 \\
\hline Respiratory system disorders & 4 & 30 & 0.89 \\
\hline Skeleto-muscular problems & 10 & 81 & 0.88 \\
\hline Circulatory disorders & 5 & 33 & 0.87 \\
\hline Cut and wounds & 4 & 23 & 0.86 \\
\hline Genito-urological problems & 4 & 22 & 0.85 \\
\hline Fever, headache, malaria & 11 & 65 & 0.81 \\
\hline ENT problems & 11 & 70 & 0.76 \\
\hline Cough-cold & 10 & 39 & 0.76 \\
\hline Oral and dental problems & 6 & 22 & 0.6 \\
\hline Others & 3 & 6 &
\end{tabular}

Table 3. Fidelity values of most frequently used medicinal plants used against a given disease category.

\begin{tabular}{llllc}
\hline Disease categories & Medicinal plant & $\boldsymbol{N}_{\boldsymbol{p}}$ & $\boldsymbol{N}$ & $\boldsymbol{F L}$ value (\%) \\
\hline \multirow{2}{*}{ Gastro-intestinal disorders } & Begonia picta & 12 & 12 & 100 \\
& Aconitum palmatum & 12 & 12 & 100 \\
\hline ENT problems & Clematis buchananiana & 11 & 12 & 91.6 \\
\hline \multirow{4}{*}{ Skeleto-muscular problems } & Astilbe rivularis & 12 & 12 & 100 \\
& Bergenia ciliata & 12 & 12 & 100 \\
& Gonostegia hirta & 11 & 12 & 91.6 \\
& Viscum album & 11 & 12 & 91.6 \\
\hline Cough-cold & Euodia fraxinifolia & 10 & 12 & 83.3 \\
\hline Fever, headache, malaria & Swertia chirayita & 12 & 12 & 100 \\
\hline \multirow{2}{*}{ Circulatory disorders } & Nasturtium officinale & 9 & 12 & 75 \\
\hline Cut and wounds & Swertia chirayita & 8 & 12 & 66 \\
\hline Oral and dental problems & Galium asperifolium & 8 & 12 & 66 \\
\hline Respiratory system disorders & Zanthoxylum oxyphyllum & 7 & 12 & 58 \\
\hline Dermatological disorders & Drymaria cordata & 12 & 12 & 100 \\
\hline Genito-urological problems & Remusatia pumila & 12 & 12 & 100 \\
\hline Others (heat illness) & Macropanax undulatus & 8 & 12 & 66 \\
\hline
\end{tabular}

Table 4. Average use reports categorized by age, gender and occupation of informants.

\begin{tabular}{|c|c|c|c|c|c|c|c|c|}
\hline \multirow[b]{2}{*}{ Disease categories } & \multicolumn{3}{|c|}{ Age group } & \multicolumn{2}{|c|}{ Gender } & \multicolumn{3}{|c|}{ Occupation } \\
\hline & $20-40$ & $40-60$ & $60+$ & Male & Female & Teacher & Farmer & $\begin{array}{c}\text { Traditional } \\
\text { healer }\end{array}$ \\
\hline Gastrointestinal disorders & 7.8 & 13 & 20 & 11.5 & 10.8 & 7.6 & 10.1 & 20 \\
\hline ENT problems & 5.5 & 5.3 & 7.5 & 5.8 & 5.8 & 5.6 & 5.4 & 7.5 \\
\hline Fever, headache, malaria & 4.8 & 5 & 8 & 4.8 & 6 & 5.6 & 4.6 & 8 \\
\hline $\begin{array}{l}\text { Skeleto-muscular } \\
\text { problems }\end{array}$ & 5.8 & 8.6 & 7.5 & 7.1 & 6.5 & 3.2 & 7.2 & 7.5 \\
\hline Cough-cold & 3.8 & 2.6 & 2 & 2.8 & 3.6 & 4.3 & 3.1 & 2 \\
\hline Oral and dental problems & 1.2 & 3 & 2 & 2.1 & 1.5 & 0.6 & 2.28 & 2 \\
\hline Circulatory disorders & 2.85 & 2.66 & 2.5 & 3 & 2.6 & 2.6 & 2.85 & 2.5 \\
\hline Cut and wounds & 1.57 & 3 & 1 & 2 & 1.4 & 1 & 2.4 & 1 \\
\hline $\begin{array}{l}\text { Genito-urological } \\
\text { problems }\end{array}$ & 1.8 & 2.3 & 1 & 1.3 & 2.3 & 2 & 2 & 1 \\
\hline $\begin{array}{l}\text { Respiratory system } \\
\text { disorders }\end{array}$ & 2.28 & 3 & 2.5 & 2.3 & 2.8 & 2.6 & 2.4 & 2.5 \\
\hline Dermatological disorders & 2.57 & 3 & 2.5 & 2.8 & 2.5 & 2.6 & 2.7 & 2.5 \\
\hline Other ailments & 0.1 & 0.66 & 2 & 0.3 & 0.6 & 0 & 0.28 & 2 \\
\hline Total (Percentage) & $\begin{array}{l}40.07 \\
(27 \%) \\
\end{array}$ & $\begin{array}{l}52.12 \\
(34 \%)\end{array}$ & $\begin{array}{c}58.5 \\
(39 \%)\end{array}$ & $\begin{array}{c}45.8 \\
(49.6 \%)\end{array}$ & $\begin{array}{c}46.4 \\
(50.3 \%)\end{array}$ & $\begin{array}{c}37.7 \\
(27 \%)\end{array}$ & $\begin{array}{c}45.3 \\
(32 \%) \\
\end{array}$ & $58.5(41 \%)$ \\
\hline
\end{tabular}


economic benefits to poor and marginalized community (Bhattarai, 2017). It is also important to have participatory management, education and awareness programs that will help to optimize the benefits of the medicinal plants sector in this area (Bhattarai and Khadka, 2016).

\section{Conclusion}

The present study reveals that some people of Thami community have immense knowledge regarding the use of medicinal plants and rely on them for treatment of various kinds of diseases. Many species used as medicine are under threatened due to more extensive use, over grazing, habitat destruction, high preference or rare existence. Hence, there is need for conservation of valuable medicinal plant species and also the young generations should be trained to acquire the knowledge which will otherwise get extinct.

\section{Acknowledgements}

I am grateful to the Department of Plant Resources, Thapathali, Nepal for necessary funding. I would like to thank Mr. Bhim Bahadur Thami, Mrs. Gau Maya Thami, Mrs. Kumari Thami and all the local people of the study area who helped in this study. Similarly, Mr. Dipen Bam, Mr. Yam Kafle and Padam Prasad Acharya of District Plant Research Centre, Ilam are highly acknowledged for their kind cooperation for questionnaire fill up and herbarium management. I am grateful to Dr. Maan Bahadur Rokaya for his immense help and valuable suggestions prior to the field study, questionnaire preparation, data collection and data analysis. Thank is also due to Mr. Madan K. Khadka for providing GIS map of the study area.

\section{References}

Bajpai, O., J. Pandey and L.B. Chaudhary 2016. Ethnomedicinal uses of tree species by Tharu tribes in the Himalayan terai region of India. Research Journal of Medicinal Plant 10(1): 19-41.

Baral, S.R. and P.P. Kurmi 2006. A compendium of medicinal plants in Nepal. Rachana Sharma, Kathmandu, Nepal.

Beltrán-Rodríguez, L., A. Ortiz-Sánchez, N.A. Mariano, B. Maldonado-Almanza and V. ReyesGarcía 2014. Factors affecting ethnobotanical knowledge in a mestizo community of the Sierra de Huautla Biosphere Reserve, Mexico. Journal of Ethnobiology and Ethnomedicine 10: 14.

Bhattarai, K.R. 2017. Ethnomedicinal practices of the Lepcha community in Ilam, east Nepal. Journal of Plant Resources 15(1): 31-44.
Bhattarai, K.R. and M.K. Khadka 2016. Ethnobotanical survey of medicinal plants from Ilam District, east Nepal. Our Nature 14(1): 7891.

Bhattarai, K.R. and S.K. Acharya 2015. Documentation of ethnobotanical knowledge of Tharu people on the utilization of plant resources in Gadariya and Phulwari VDCs of Kailali District, West Nepal. Bulletin of Department of Plant Resources 37: 41-50.

Bhattarai, N.K. 1989. Ethnobotanical studies in central Nepal: The ceremonial plant foods. Contribution to Nepalese Studies 16: 35-41.

Bhattarai, S and O. Basukala 2016. Antibacterial activity of selected ethnomedicinal plants of Sagarmatha region of Nepal. International Journal of Therapeutic Applications 31: 27-31.

Bhattarai, S. and R. Tamang 2017. Medicinal and aromatic plants: A synopsis of Makawanpur District, central Nepal. International Journal of Indigenous Herbs and Drugs 2(3): 6-15.

Bisht, A.K., A. Bhatt, A.S. Rawal and U. Dar 2006. Prioritization and conservation of Himalayan medicinal plants: Angelica glauca Edgew. as a case study. Ethnobotany Research and Applications 4: 011-023.

Budathoki, C.B., M. Subedi, P. Pradhan and G. Chhetri 2008. Case study of ethnic identity and socioeconomic condition of Thami in Dolakha District. Janajati Social and Economic Empowerment Project (JSEEP), Kathmandu.

CBS 2013. Statistical year book of Nepal- 2013. Government of Nepal, National Planning Commission Secretariat, Central Bureau of Statistics, Kathmandu, Nepal.

CBS 2014a. National Population and Housing Census 2011: Social characteristics tables (Cast/Ethnicity, Mother tongue and second language). Vol. 5, Part II. Government of Nepal, National Planning Commission Secretariat, Central Bureau of Statistics, Kathmandu, Nepal.

CBS 2014b. National Population and Housing Census 2011 (Village Development committee/Municipality): Ilam. Vol. 6. Government of Nepal, National Planning Commission Secretariat, Central Bureau of Statistics, Kathmandu, Nepal.

DFRS 2015. District wise forest cover map of Nepal. Forest Resource Assessment (FRA) Nepal, Department of Forest Research and Survey (DFRS). Kathmandu, Nepal.

Forman, L. and D. Bridson 1989. The herbarium handbook. Royal Botanic Gardens, Kew.

Friedman, J., Z. Yaniv, A. Dafni and D. Palevitch 1986. A preliminary classification of the healing potential of medicinal plants, based on a rational analysis of an ethnopharmacological field survey among bedouins in the Negev desert, Israel. Journal of Ethnopharmacology 16: 275-287. 
Ghimire, S.K. 2008. Sustainable harvesting and management of medicinal plants in the Nepal Himalaya: Current issues, knowledge gaps and research priorities. In Medicinal Plants of Nepal: An Anthology of Contemporary Research (Jha, P.K., S.B. Karmacharya, M.K. Chhetri, C.B. Thapa and B.B. Shrestha Eds.). Ecological Society of Nepal (ECOS), Kathmandu. pp. 25-44.

Ghimire, S.K., O. Gimenez, R. Pradel, D. McKey and Y. Aumeeruddy-Thomas 2008. Demographic variation and population viability in a threatened Himalayan medicinal and aromatic herb Nardostachys grandiflora: matrix modeling of harvesting effects in two contrasting habitats. Journal of Applied Ecology 45: 41-51

Giday, M., Z. Asfaw and Z. Woldu 2009. Medicinal plants of the Meinit ethnic group of Ethopia: An ethnobotanical study. Journal of ethnopharmacology 124(3): 513-521.

Heinrich, M., A. Ankli, B. Frei, C. Weimann and O. Sticher 1998. Medicinal plants in Mexico: healers' consensus and cultural importance. Social Science and Medicine 47(11): 1859-1871.

HMG 2002. National Foundation for Development of Indigenous Nationalities Act, 2058 (2002 A.D.). His Majesty's Government of Nepal, Department of Printing, Singha Durbar, Kathmandu.

Hoffman, B. and T. Gallaher 2007. Importance indices in ethnobotany. Ethnobotany Research and Applications 5: 201-218.

Luitel, D.R., M.B. Rokaya, B. Timsina and Z. Münzbergová 2014. Medicinal plants used by the Tamang community in the Makwanpur District of Central Nepal. Journal of Ethnobiology and Ethnomedicine 10(5).

Mahmood, A., A. Mahmood and R.N. Malik 2012. Indigenous knowledge of medicinal plants from Leepa valley, Azad Jammu and Kashmir, Pakistan. Journal of Ethnopharmacology 143: 338-346.

Manandhar, L.N. and R.P. Chaudhary 1992. Medicinal plants and their traditional use by tribal people of Saptari District, Nepal. Proceedings of $1^{\text {st }}$ National Botanical Conference, Aug.11-12, Kathmandu, Nepal.

Manandhar, N.P. 2002. Plants and people of Nepal. Timber Press, Portland, Oregon.

Merétika, A.H.C., N. Peroni and N. Hanazaki 2010. Local knowledge of medicinal plants in three artisanal fishing communities (Itapoá, Southern Brazil), according to gender, age, and urbanization. Acta Botanica Brasilica 24(2): 386394.

Phillips, O. and A.H. Gentry 1993. The useful plants of Tambopata, Peru: I. Statistical hypotheses tests with a new quantitative technique. Economic Botany 47: 15-32.

Polunin, O. and A. Stainton 1984. Flowers of the Himalaya. Oxford University Press, New Delhi, India.
Pradhan, B.K. and H.K. Badola 2008. Ethnomedicinal plant use by Lapcha tribe of Dzongu valley, bordering Khangchendzonga Biosphere Reserve, in North Sikkim, India. Journal of Ethnobiology and Ethnomedicine 4: 22.

Quinlan, M.B. and R.J. Quinlan 2007. Modernization and medicinal plant knowledge in a Caribbean Horticultural Village. Medical Anthropology Quarterly 21(2): 169-192.

Rajbhandari, S. and D. Winkler 2015. Ethnobotany. In Nepal: An introduction to the natural history, ecology and human environment of the Himalayas (Miehe, G., C.A. Pendry and R. Chaudhary Eds.). Royal Botanic Garden, Edinburgh. pp. 271-285.

Rani, S., G. Singh and N. Sood 2017. Comparison of intensity and percolation of traditional knowledge of Calotropis procera and Calotropis gigantea in rural area of Kurukshetra District, Haryana- A survey. Journal of Medicinal Plants Studies 5(2): 36-38.

Rokaya, M.B., Y. Uprety, R.C. Poudel, B. Timsina, Z. Münzbergová, H. Asselin, A. Tiwari, S.S. Shrestha and S.R. Sigdel 2014. Traditional uses of medicinal plants in gastrointestinal disorders in Nepal. Journal of Ethnopharmacology 158: 221229.

Rokaya, M.B., Z. Münzbergová and B. Timsina 2010. Ethnobotanical study of medicinal plants from the Humla District of western Nepal. Journal of Ethnopharmacology 130: 485-504.

Sarwar, M., I.D. Attitalla and M. Abdollahi 2011. A review on the recent advances in pharmacological studies on medicinal plants; Animal studies are done but clinical studies needs completing. Asian Journal of Animal and Veterinary Advances 6: 867-883.

Saslis-Lagoudakis, C.H., J.A. Hawkins, S.J. Greenhill, C.A. Pendry, M.F. Watson, W. Tuladhar-Douglas, S.R. Baral and V. Savolainen 2014. The evolution of traditional knowledge: environment shapes medicinal plant use in Nepal. Proceedings of the Royal Society B 281: 20132768. DOI: 10.1098/rspb.2013.2768.

Shneiderman, S. and M. Turin 2000. Thangmi, Thami, Thani? Remembering forgotten people. Niko Bacinte: 82-100.

Shneiderman, S. and M. Turin 2006. Revisiting ethnography, recognizing a forgotten people: The Thangmi of Nepal and India. Studies in Nepali History and Society 11(1): 97-181.

Shrestha, K. 1998. Dictionary of Nepalese plant names. Mandala Book Point, Kantipath, Kathmandu, Nepal.

Shrestha, N., D. Prasai, K.K. Shrestha and X. Zhang 2014. Ethnomedicinal practices in the highlands of central Nepal: A case study of Syaphru and Langtang village in Rasuwa District. Journal of ethnopharmacology 155(2): 1204-1213.

Singh, A.G., A. Kumar and D.D. Tiwari 2012. An ethnobotanical survey of medicinal plants used in 
Terai forest of western Nepal. Journal of Ethnobiology and Ethnomedicine 8: 19.

Singh, A.G., A. Kumar, D.D. Tiwari and K.A. Bharati 2018. New ethnomedicinal claims from Magar community of Palpa District, Nepal. Indian journal of traditional knowledge 17(3): 499-511.

Srithi, K., H. Balslevb, P. Wangpakapattanawonga, P. Srisangac and C. Trisonthia 2009. Medicinal plant knowledge and its erosion among the Mien (Yao) in northern Thailand. Journal of Ethnopharmacology 123: 335-342.

Stainton, A. 1988. Flowers of the Himalaya: A supplement. Oxford University Press, New Delhi, India.

Subba, B., C. Srivastav and R.C. Kandel 2016. Scientific validation of medicinal plants used by Yakkha community of Chanuwa VDC, Dhankuta, Nepal. Springer Plus 5: 155.

Tamang, P. and N.B. Singh 2014. Medical ethnobiology and indigenous knowledge system of the Lapcha of Fikkal VDC of Ilam, Nepal. Journal of Institute of Science and Technology 19(2): 4552.

Thami, S.K. 2017. Know about Thami (Text: Nepali). Retrieved on September 5, 2018, from: http://bhalakusari.com/2017/3/111

Thapa, L.B., T.M. Dhakal, R. Chaudhary and H. Thapa 2013. Medicinal plants used by Raji ethnic tribe of Nepal in treatment of gastrointestinal disorders. Our Nature 11(2): 177-186.
Toledo, B.A., L. Galetto and S. Colantonio 2009. Ethnobotanical knowledge in rural communities of Cordoba (Argentina): the importance of cultural and biogeographical factors. Journal of Ethnobiology and Ethnomedicine 5: 40.

Torres-Avilez, W., P. Muniz de Medeiros and U.P. Albuquerque 2016. Effect of gender on the knowledge of medicinal plants: Systematic review and meta-analysis. Evidence-Based Complementary and Alternative Medicine. Article ID 6592363,13p.DOI: http://dx.doi.org/10.1155/2016 16592363

Turin, M. 2003. Ethnobotanical notes on Thangmi plant names and their medicinal and ritual uses. Contributions to Nepalese Studies 30(1): 19-52.

Turin, M. 2004. Newar-Thangmi lexical correspondences and the linguistic classification of Thangmi, Journal of Asian and African studies 68: $97-120$.

Uprety, Y., H. Asselin, E.K. Boon, S. Yadav and K.K. Shrestha 2010. Indigenous use and bioefficacy of medicinal plants in the Rasuwa District, Central Nepal. Journal of ethnobiology and ethnomedicine 6: 3 .

URL 2018a. https://en.wikipedia.org/wiki/Thami (Retrieved on September 5, 2018)

URL2018b. https://joshuaproject.net/people_groups/ 15461/NP (Retrieved on September 5, 2018)

WHO 2002. World Health Organization traditional medicine strategy 2002-2005. WHO, Geneva. 
Bhattarai / Our Nature (2018), 16 (1): 55-67 